\title{
Risk factors and outcomes of acute kidney injury in South African critically ill adults: a prospective cohort study
}

\author{
Ryan E. Aylward ${ }^{1 *}\left(\mathbb{D}\right.$, Elizabeth van der Merwe ${ }^{1,2}$, Sisa Pazi ${ }^{3}$, Minette van Niekerk', Jason Ensor ${ }^{4,5}$,
} Debbie Baker ${ }^{1,2}$ and Robert J. Freercks ${ }^{4,5}$

\begin{abstract}
Background: There is a marked paucity of data concerning AKI in Sub-Saharan Africa, where there is a substantial burden of trauma and HIV.

Methods: Prospective data was collected on all patients admitted to a multi-disciplinary ICU in South Africa during 2017. Development of AKI (before or during ICU admission) was recorded and renal recovery 90 days after ICU discharge was determined.

Results: Of 849 admissions, the mean age was 42.5 years and mean SAPS 3 score was 48.1. Comorbidities included hypertension (30.5\%), HIV (32.6\%), diabetes (13.3\%), CKD (7.8\%) and active tuberculosis (6.2\%). The most common reason for admission was trauma (26\%). AKI developed in 497 (58.5\%). Male gender, illness severity, length of stay, vasopressor drugs and sepsis were independently associated with AKI. AKI was associated with a higher in-hospital mortality rate of $31.8 \%$ vs $7.23 \%$ in those without AKI. Age, active tuberculosis, higher SAPS 3 score, mechanical ventilation, vasopressor support and sepsis were associated with an increased adjusted odds ratio for death. HIV was not independently associated with AKI or hospital mortality. CKD developed in 14 of 110 (12.7\%) patients with stage $3 \mathrm{AKl}$; none were dialysis-dependent.

Conclusions: In this large prospective multidisciplinary ICU cohort of younger patients, AKI was common, often associated with trauma in addition to traditional risk factors and was associated with good functional renal recovery at 90 days in most survivors. Although the HIV prevalence was high and associated with higher mortality, this was related to the severity of illness and not to HIV status per se.
\end{abstract}

Keywords: AKI, Africa, HIV, ICU, Dialysis

\section{Background}

Acute Kidney Injury (AKI) is commonly encountered in the Intensive Care Unit (ICU) [1], but with a widely variable reported incidence due to non-standardization of its definition [2]. Regardless of the definition used, AKI is a well-recognized independent risk factor for mortality, is associated with substantial morbidity and is a current major cause for global concern [3-6]. Furthermore, AKI requiring dialysis is now recognized as a risk factor for end stage kidney disease in the long term $[7,8]$ and is

\footnotetext{
* Correspondence: ryaylion@gmail.com

${ }^{1}$ Adult Critical Care Unit, Livingstone Hospital, Port Elizabeth, South Africa Full list of author information is available at the end of the article
}

associated with poor long-term quality of life after ICU discharge $[9,10]$.

AKI has been well characterized in high income (HI) countries and appears to be increasing in incidence [5, 11-13]. However, there is a marked paucity of data from African ICU's concerning the incidence, aetiology and effect of AKI on mortality and functional renal recovery, where the prevalence of HIV and trauma is high and where resources are often limited $[6,14,15]$. Renal replacement therapy is an expensive [16] and scarce resource in South Africa [17] and in particular, in the rest of sub-Saharan Africa $[18,19]$.

The International Society of Nephrology has boldly called for 0by25: The elimination of preventable deaths

(C) The Author(s). 2019 Open Access This article is distributed under the terms of the Creative Commons Attribution 4.0 International License (http://creativecommons.org/licenses/by/4.0/), which permits unrestricted use, distribution, and 
from AKI by 2025 [20]. Timely diagnosis and prevention remain the most important strategies. Accordingly, understanding the epidemiology of AKI in lower and middle income (LMI) countries must be a key step in tackling this problem. Our aim therefore, was to describe the epidemiology of AKI in patients admitted to our South African multidisciplinary ICU, where there is currently a high prevalence of HIV and traumaassociated admissions. We sought to characterize the factors associated with the development of AKI, the effect of HIV on AKI as well as survival and to report on the 90-day renal function outcome of all who developed AKI.

\section{Methods}

\section{Study design and setting}

An observational prospective design was used. Cohorts were divided into those who did and did not develop AKI (prior to and/or after admission to the ICU) as well as by HIV status where known. All patients older than 12 years admitted to the Livingstone Hospital ICU between 3 January 2017 and 3 January 2018 were included. Patients who died within $6 \mathrm{~h}$ of being admitted to ICU, those who were brain-dead awaiting organ harvesting, and patients with known or presumed end stage kidney disease were excluded from the study.

The Livingstone Hospital adult ICU is a tertiary service, closed, multi-disciplinary 16 -bed unit serving a catchment area of 1.6 million people. The hospital is government-funded and is located in the Nelson Mandela Bay Metropole in South Africa where 1.15 million people live in an urban setting, $12.3 \%$ of whom live in informal shack dwellings and $36.6 \%$ of whom are unemployed. The balance of 450,000 people live in surrounding rural areas within a radius of $250 \mathrm{~km}$ [21]. Full time consultant supervision is provided by two intensivists and two nephrologists. The provision of dialysis is also government-funded and not restricted within the ICU or by HIV status. However, general prognosis and current resource limitations are taken into consideration prior to the admission of any patient to the ICU [22]. The modality of dialysis is chosen by the treating consultant based on clinical status with a preference for intermittent haemodialysis or sustained low efficiency daily dialysis (SLEDD) due to cost constraints. Continuous renal replacement therapy (CRRT) is reserved for severely haemodynamically unstable patients and for those with raised intracranial pressure. Referring disciplines include medicine, trauma, general surgery, urology, neurosurgery, orthopaedics, obstetrics and gynaecology. Elective cardiology and cardio-thoracic surgery have their own dedicated ICU. Obstetrics also has their own high care although patients with advanced organ dysfunction are referred to our unit.

\section{Definition of acute kidney injury}

AKI was diagnosed and staged according to the Kidney Diseases Improving Global Outcomes (KDIGO) definition [23]. A normal serum creatinine not older than 90 days was assumed to be the baseline where available, as recommended [24]. The cause of AKI was determined by the treating intensivist/nephrologist and more than one cause could be assigned.

\section{Definition of outcomes}

AKI was recorded as resolved once creatinine improved to the known or presumed baseline. If renal function had not recovered by hospital discharge, patients were followed up for at least 90 days following ICU discharge or until renal recovery. Patients who had not recovered their renal function by this time were deemed to have chronic kidney disease (CKD).

\section{Ethical approval}

Approval for the study (protocol number: 067/2016) was granted by the Walter Sisulu University Human Research Ethics Unit. Since we were conducting a nonexperimental study that would not influence clinical decision-making or patient management, the need for study participant consent was waived by the Ethics Unit.

\section{Data collection and management}

Demographic data including age, sex, race and details related to co-morbidities were recorded. For patients known with HIV, a premorbid CD4 count and viral load was recorded, where available. The Simplified Acute Physiology Score 3 (SAPS 3) [25] was calculated within the first hour of ICU admission. The Sequential Organ Failure Assessment (SOFA) [26] was calculated 24h after admission and every third day thereafter, or sooner if the patient's condition deteriorated. Vasopressor and mechanical ventilation requirements were also recorded. Cause of AKI, renal replacement modality and creatinine on admission, peak and discharge were recorded for patients in the AKI cohort. Sepsis was defined using Sepsis-3 criteria [27].

\section{Statistical analysis}

Data were exported from the Research Electronic Data Capture (REDCap) hosted at the University of Cape Town [28] and analyzed with RStudio, 2017 (Version 3.4.2). Hypothesis tests were considered significant if the two-sided $p$-value $<0.05$. Continuous data were tested for normality using the Kolmogorov-Smirnov, ShapiroWilk, Anderson-Darling and Pearson's chi-squared tests. Normally distributed data are reported as means (standard deviation) and skewed data as medians (interquartile range). Discrete data are presented as numbers (percentages). The student's t-test and the Mann-Whiteney $U$ 
test were used to compare continuous data and the Chisquare and Fischer's exact test were used for discrete data, as appropriate. Missing outcome data $(n=12)$ were analysed using multiple imputation. Hazard ratios for mortality by AKI and HIV status were calculated using the Cox proportional hazards model. Multivariate logistic-regression models were used to determine associations of developing AKI and dying. Variables by bivariate analysis with an alpha level $<0.1$ between AKI and non-AKI cohorts as well as known predictors for AKI (age, sex, hypertension, active malignancy and admission SAPS 3 score) were included in the model. KDIGO stages 1, 2 and 3 were compared to patients who did not develop AKI as reference.

\section{Results}

A total of 875 patients were admitted to the ICU during the study period and 26 were excluded from the analysis; Fig. 1 details the reasons for exclusion. Vital status after ICU discharge could not be established for 12 patients due to in-patient transfers to other hospitals and the unavailability of further records; six were in the AKI cohort, and outcomes were imputed.

In-hospital mortality was $21.6 \%$ while mortality in ICU was $13.4 \%$. The most common diagnoses admitted were: Assault (14\%), motor and pedestrian vehicle accidents (12\%), acute abdomen (10\%), pneumonia (6\%; including cases later identified as tuberculosis) and self-inflicted drug/toxin overdose (3.5\%). Admissions included surgical emergencies $(n=451,53.1 \%)$, medical emergencies $(n=261,30.7 \%)$, surgical elective cases $(n=110,13 \%)$ and obstetric emergencies $(n=27,3.2 \%)$. Table 1 shows the baseline characteristics of all patients admitted to the ICU and by AKI status.

\section{Severity and causes of AKI}

AKI developed in 497 patients (58.5\%), 80.5\% of whom were diagnosed with AKI on admission to the ICU. The maximum KDIGO stage was stage 1 in 138 (27.7\%), stage 2 in $152(30.6 \%)$ and stage 3 in 207 (41.7\%) patients respectively.

Figure 2 shows the main causes identified for developing AKI. Herbal ingestion was only documented in 4 patients and 12 patients were exposed to antituberculous drugs (rifampicin and isoniazid). Of the 105 HIV positive patients who developed AKI, 24 had received tenofovir prior to AKI diagnosis. Histology was obtained in situations where the cause of AKI was not clear or the clinical course of the patient was unclear. Kidney biopsies were performed in 5 patients ( $1 \%$ of the AKI cohort), only 1 of whom had HIV which showed interstitial HIV associated nephropathy. The others were in HIV negative subjects; 3 showed features of mesangiocapillary glomerulonephritis, 1 of which was crescentic and 1 had features of ascending pyelonephritis.

\section{Risk factors associated with AKI}

Risk factors associated with AKI are presented in Table 2. On multivariate analysis, male gender, increased SOFA and/or SAPS 3 score, increased length of stay, the need for vasopressor drugs and

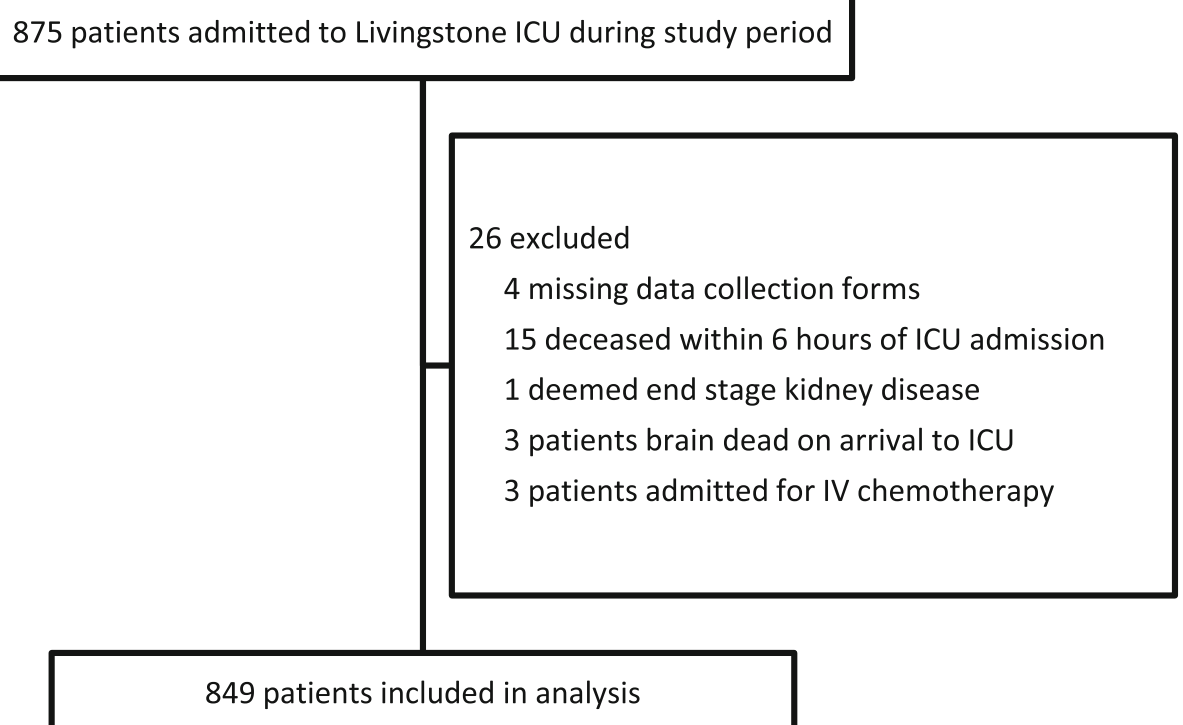

Fig. 1 Diagram of patients included in the analysis 
Table 1 Baseline characteristics of all patients admitted to the ICU

\begin{tabular}{|c|c|c|c|c|}
\hline Characteristic & All patients $n=849$ & $\mathrm{AKI} n=497$ & No AKI $n=352$ & $p$-value \\
\hline \multicolumn{5}{|l|}{ Demographics } \\
\hline Mean age, years ${ }^{a}(S D)$ & $42.5(16.8)$ & $43.7(16.8)$ & 40.9 (16.6) & 0.017 \\
\hline Male gender $\%$ & 58.9 & 61.2 & 55.7 & 0.110 \\
\hline \multicolumn{5}{|l|}{ Race $^{a} \%$} \\
\hline Black African & 58.0 & 60.5 & 54.3 & 0.067 \\
\hline Mixed Ancestry & 27.4 & 26.0 & 29.6 & 0.248 \\
\hline BCaucasian & 13.8 & 12.7 & 15.3 & 0.267 \\
\hline Other & 0.8 & 0.8 & 0.8 & 0.827 \\
\hline \multicolumn{5}{|l|}{ Co-morbidities } \\
\hline Diabetes $^{\mathrm{a}} \%$ & 13.2 & 16.1 & 9.1 & 0.003 \\
\hline Mean HbA1c \% (SD) & $9.9(3.2)$ & $10.3(3.1)$ & $9.1(3.3)$ & 0.142 \\
\hline Hypertension ${ }^{a} \%$ & 31.6 & 32.2 & 30.7 & 0.641 \\
\hline Ischaemic heart disease \% & 4.2 & 4.6 & 3.7 & 0.506 \\
\hline Active Tuberculosis \% & 6.1 & 6.6 & 5.4 & 0.457 \\
\hline Chronic kidney disease $\%$ & 7.7 & 6.4 & 9.4 & 0.113 \\
\hline Mean CKD eGFR (SD) & $32(19)$ & $40(16)$ & $21(1)$ & $<0.001$ \\
\hline Epilepsy \% & 4.8 & 5.0 & 4.6 & 0.746 \\
\hline Malignancy $\%$ & 3.3 & 3.6 & 2.8 & 0.530 \\
\hline \multicolumn{5}{|l|}{ HIV } \\
\hline \multicolumn{5}{|l|}{ Known status, $n=472$ (56.1\%) } \\
\hline Positive, $n$ (\%) & $155(32.6)$ & $105(35.1)$ & $50(28.3)$ & 0.010 \\
\hline Negative, $n(\%)$ & $321(67.4)$ & $194(64.9)$ & $127(71.8)$ & 0.382 \\
\hline \multicolumn{5}{|l|}{ In HIV positive cohort: } \\
\hline Median CD4 count, cells/uL (IQR) & $318(156-493)$ & $205(119-391)$ & $404(308-513)$ & 0.025 \\
\hline Median viral load, log (IQR) & $2.0(1.98-4.04)$ & $2.0(1.88-3.62)$ & $2.5(2.00-4.64)$ & 0.234 \\
\hline Receiving HAART ${ }^{a} \%$ & 11.9 & 14.5 & 8.2 & 0.006 \\
\hline \multicolumn{5}{|l|}{ Severity of illness } \\
\hline Emergency admissions \% & 87.0 & 92.8 & 79.0 & $<0.001$ \\
\hline Mean SAPS 3 score ${ }^{a}$ (SD) & 48.1 & 54.0 & 39.7 & $<0.001$ \\
\hline Median highest SOFA score ${ }^{a}(\mathrm{QQR})$ & $4(1-6)$ & $6(3-9)$ & $2(1-4)$ & $<0.001$ \\
\hline Sepsis and septic shock $\%$ & 30.2 & 43.1 & 11.9 & $<0.001$ \\
\hline Ventilated $\%$ & 53.7 & 64.8 & 38.2 & $<0.001$ \\
\hline median ventilator days (IQR) & $3(1-7)$ & $3(1-8)$ & $2(1-4)$ & $<0.001$ \\
\hline Required vasopressors ${ }^{\mathrm{a}} \%$ & 25.0 & 38.8 & 5.4 & $<0.001$ \\
\hline $\operatorname{ARDS}^{\mathrm{a}} \%$ & 4.4 & 6.8 & 0.9 & $<0.001$ \\
\hline Median ICU days (IQR) & $3(1-6)$ & $4(2-8)$ & $2(1-4)$ & $<0.001$ \\
\hline ICU length of stay $>7$ days $\%$ & 24.7 & 33.2 & 12.8 & $<0.001$ \\
\hline Deceased in ICU \% & 13.0 & 20.5 & 2.3 & $<0.001$ \\
\hline
\end{tabular}

Abbreviations: AKI Acute Kidney Injury, CI Confidence interval, SD Standard deviation, IQR Interquartile range, CKD Chronic Kidney Disease, eGFR Estimated Glomerular Filtration Rate, HAART Highly Active Antiretroviral Therapy, SAPS 3 Simplified Acute Physiology Score 3, SOFA Sequential Organ Failure Assessment, ARDS Acute Respiratory Distress Syndrome. ${ }^{a}$ Variables included in multivariate analysis

the development of sepsis or septic shock were independently associated with the development of AKI by logistic-regression modeling. HIV was not independently associated with AKI.

\section{Use of renal replacement}

Eighty-eight patients were dialyzed $(42.5 \%$ of patients with stage $3 \mathrm{AKI}, 18.0 \%$ of the cohort with AKI and $10.4 \%$ of the entire cohort), $67.0 \%$ of whom were 


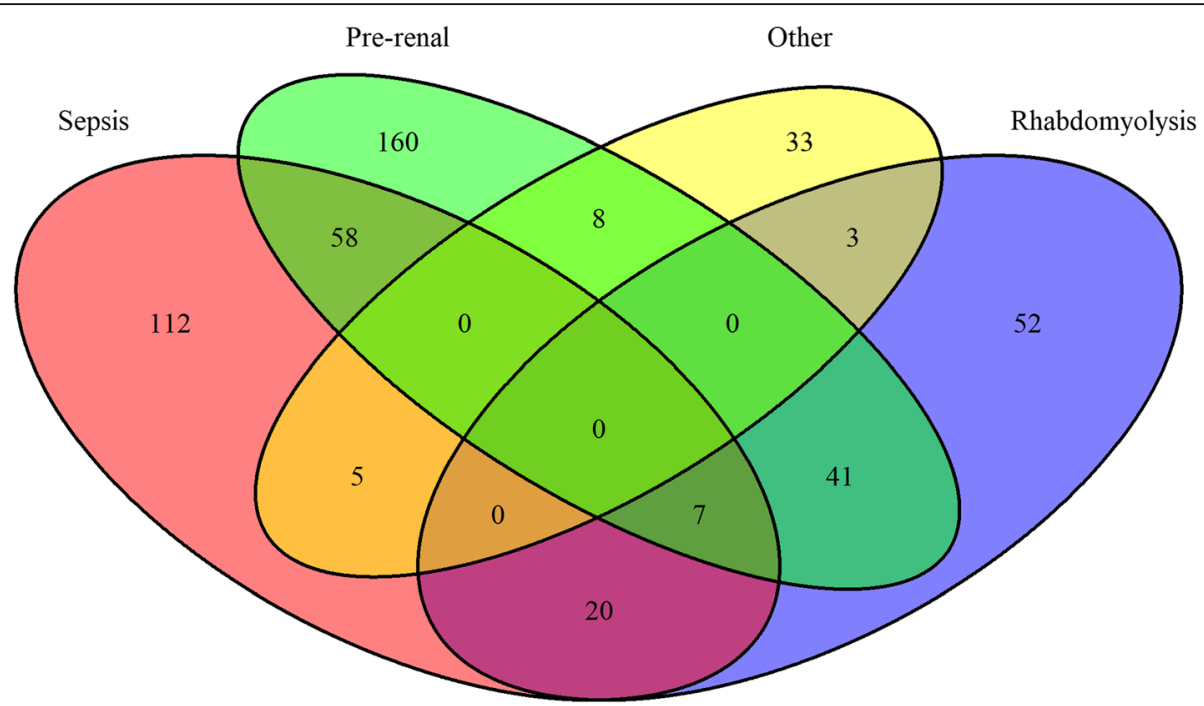

Fig. 2 Identifiable causes of AKI. A pre-renal aetiology (such as hypovolaemic shock) was identified in 55.1\%, sepsis in 40.6\% and rhabdomyolysis, usually related to trauma, in $24.8 \%$. "Other" included obstructive uropathy, direct drug nephrotoxicity, hypertensive crisis, thrombotic microangiopathy, and rapidly progressive glomerulonephritis. More than one cause may have been implicated in the same patient, especially if the patient developed more than one episode of AKI during the same admission

initiated within $24 \mathrm{~h}$ of arrival to ICU. Intermittent haemodialysis was used in most (80.7\%; Fig. 3). The most common indications for dialysis initiation were life-threatening hyperkalaemia (44.3\%), uraemic symptoms such as encephalopathy or seizures $(34.1 \%)$ and refractory metabolic acidosis (34.1\%).

\section{Mortality and AKI}

The development of AKI was associated with a higher in-hospital mortality rate of $31.8 \%$ compared to $7.2 \%$ in those without AKI (Hazards Ratio 4.07, 95\% CI 2.66;
6.21; logrank $p<0.001$ (Fig. 4)). Further, the odds of dying increased stepwise with increasing KDIGO AKI stage (Fig. 5). The absolute in-hospital mortality rate was 29/134 (21.6\%), 39/150 (26.0\%) and 87/206 (42.2\%) in stage 1, 2 and 3 AKI respectively and 32/88 (36.4\%) for those who received dialysis. In those receiving exclusively intermittent haemodialysis, mortality was $31.0 \%$ compared to 55.5 and $62.5 \%$ for those who received exclusively SLEDD or CRRT, respectively [adjusted OR (95\%CI) for CRRT: 6.59 (1.55; 27.88); $p$-value 0.01].

Table 2 Crude and Adjusted odds ratios determined by multivariate analysis exploring risk factors associated with AKI

\begin{tabular}{|c|c|c|c|}
\hline & Crude odds ratio $(95 \% \mathrm{Cl})$ & Adjusted odds ratio $(95 \% \mathrm{Cl})$ & $p$-value \\
\hline Age, per 10 year increase & $1.10(1.002 ; 1.018)$ & $1.11(0.999 ; 1.260)$ & 0.079 \\
\hline Male gender & $1.22(0.92 ; 1.608)$ & $1.44(1.008 ; 2.058)$ & 0.045 \\
\hline Diabetes & $1.88(1.228 ; 2.945)$ & $1.69(0.982 ; 2.947)$ & 0.059 \\
\hline Baseline CKD & $0.64(0.382 ; 1.066)$ & $0.32(0.163 ; 0.634)$ & 0.001 \\
\hline Receiving HAART & $1.95(1.245 ; 3.137)$ & $1.64(0.940 ; 2.920)$ & 0.086 \\
\hline Admission SOFA score & $1.48(1.391 ; 1.585)$ & $1.33(1.215 ; 1.456)$ & $<0.001$ \\
\hline SAPS 3 score & $1.07(1.062 ; 1.088)$ & $1.03(1.013 ; 1.047)$ & $<0.001$ \\
\hline ICU length of stay, days & $1.12(1.081 ; 1.155)$ & $1.04(1.007 ; 1.072)$ & 0.026 \\
\hline Required mechanical ventilation & $3.05(2.299 ; 4.072)$ & $0.56(0.359 ; 0.860)$ & 0.003 \\
\hline Required vasopressors & $11.15(6.954 ; 18.878)$ & $2.52(1.392 ; 4.700)$ & 0.003 \\
\hline Developed ARDS & $12.39(3.734 ; 76.752)$ & $3.20(0.840 ; 21.071)$ & 0.137 \\
\hline Developed sepsis or septic shock & $5.50(3.839 ; 8.038)$ & $1.81(1.119 ; 2.929)$ & 0.016 \\
\hline HIV Infection ${ }^{a}$ & $1.42(0.952 ; 2.15)$ & $0.60(0.272 ; 1.305)$ & 0.198 \\
\hline
\end{tabular}

Abbreviations: HAART Highly Active Antiretroviral Therapy, SOFA Sequential Organ Failure Assessment, SAPS 3 Simplified Acute Physiology Score 3, ARDS Acute Respiratory Distress Syndrome. ${ }^{a}$ Analysis restricted to only those with a known HIV serostatus $(n=472)$ 


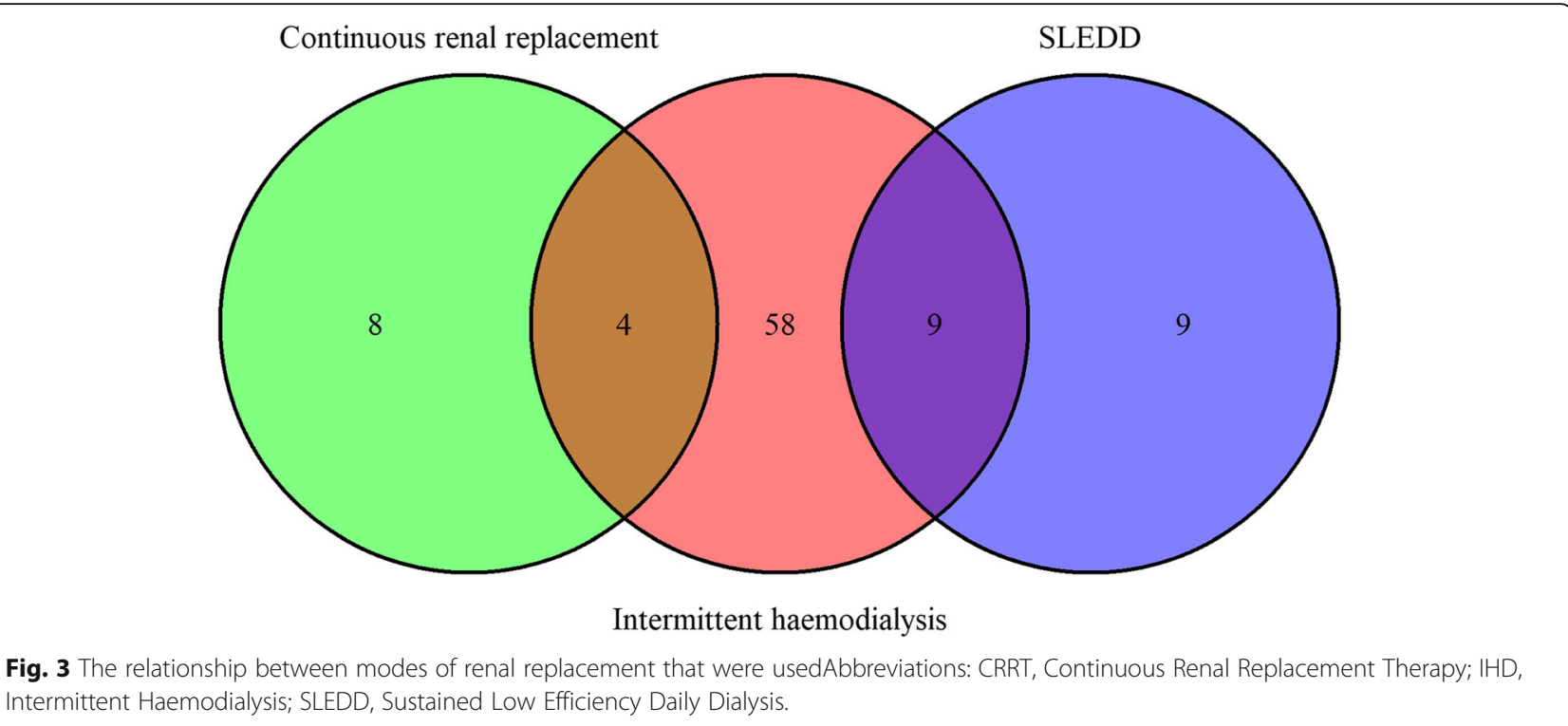

In the AKI cohort, an increased adjusted odds ratio for death was observed with increasing age, active tuberculosis, higher SAPS score, receipt of mechanical ventilation, receipt of vasopressor support and in those with sepsis (Table 3).

HIV infection was associated with worse survival in those with AKI (Fig. 6). However, on multivariate analysis, HIV was not found to be an independent risk factor for mortality (Table 3). Patients with HIV and AKI were more severely ill on admission with mean SAPS 3 score (SD) of 58.2 (15.9) versus 53.3 (13.8), $p=0.007$ and SOFA (IQR) of $8(4 ; 11)$ versus $6(3 ; 9), p=0.01$.
They also had a higher likelihood of having active tuberculosis [OR $(95 \% \mathrm{CI})=3.55$ (1.42; 9.36), $p=0.008$ ]. The median premorbid CD4 count was significantly lower in those with AKI than without (204.5 cells/microliter versus 404 cells/microliter, $p=0.025$ ) but viral suppression was similar in both groups (viral load log 2.0 vs 2.5 respectively, $p=0.234$ ). Although ICU length of stay was less in HIV patients with AKI (median ICU days 3 vs 6, $p=0.002$ ), the proportion of patients with HIV who died within the first $24 \mathrm{~h}$ was higher at $10.5 \%$ compared to $2.6 \%$ in those who were HIV negative, $p=0.009$.

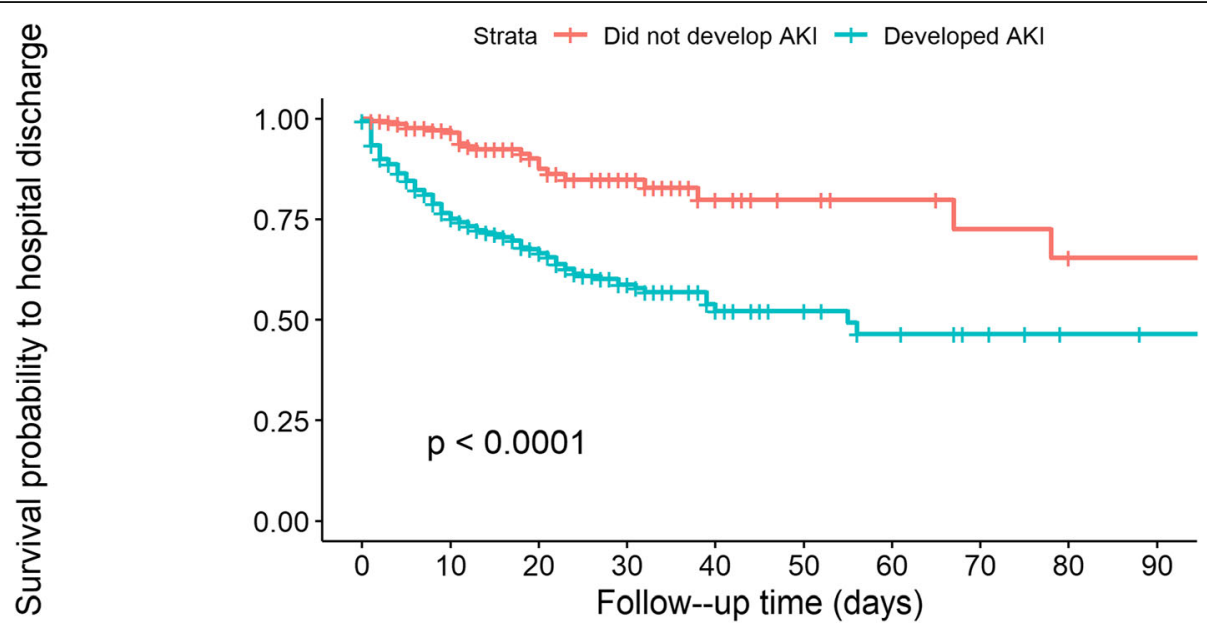

Number at risk

\begin{tabular}{|c|c|c|c|c|c|c|c|c|c|c|}
\hline \multirow{2}{*}{ 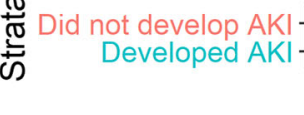 } & $\begin{array}{l}346 \\
491 \\
\end{array}$ & $\begin{array}{l}162 \\
277 \\
\end{array}$ & $\begin{array}{c}72 \\
139 \\
\end{array}$ & $\begin{array}{l}42 \\
74\end{array}$ & $\begin{array}{l}26 \\
33 \\
\end{array}$ & $\begin{array}{l}14 \\
21 \\
\end{array}$ & $\begin{array}{l}12 \\
15\end{array}$ & $\begin{array}{c}10 \\
9\end{array}$ & $\begin{array}{l}9 \\
5 \\
\end{array}$ & $\begin{array}{l}8 \\
4 \\
\end{array}$ \\
\hline & 0 & 10 & 20 & 30 & 40 & 50 & 60 & 70 & 80 & 90 \\
\hline
\end{tabular}

Fig. 4 Kaplan Meier plot showing survival probability for patients following ICU admission who developed AKI and those that did not 


\section{Odds ratios per KDIGO stage}

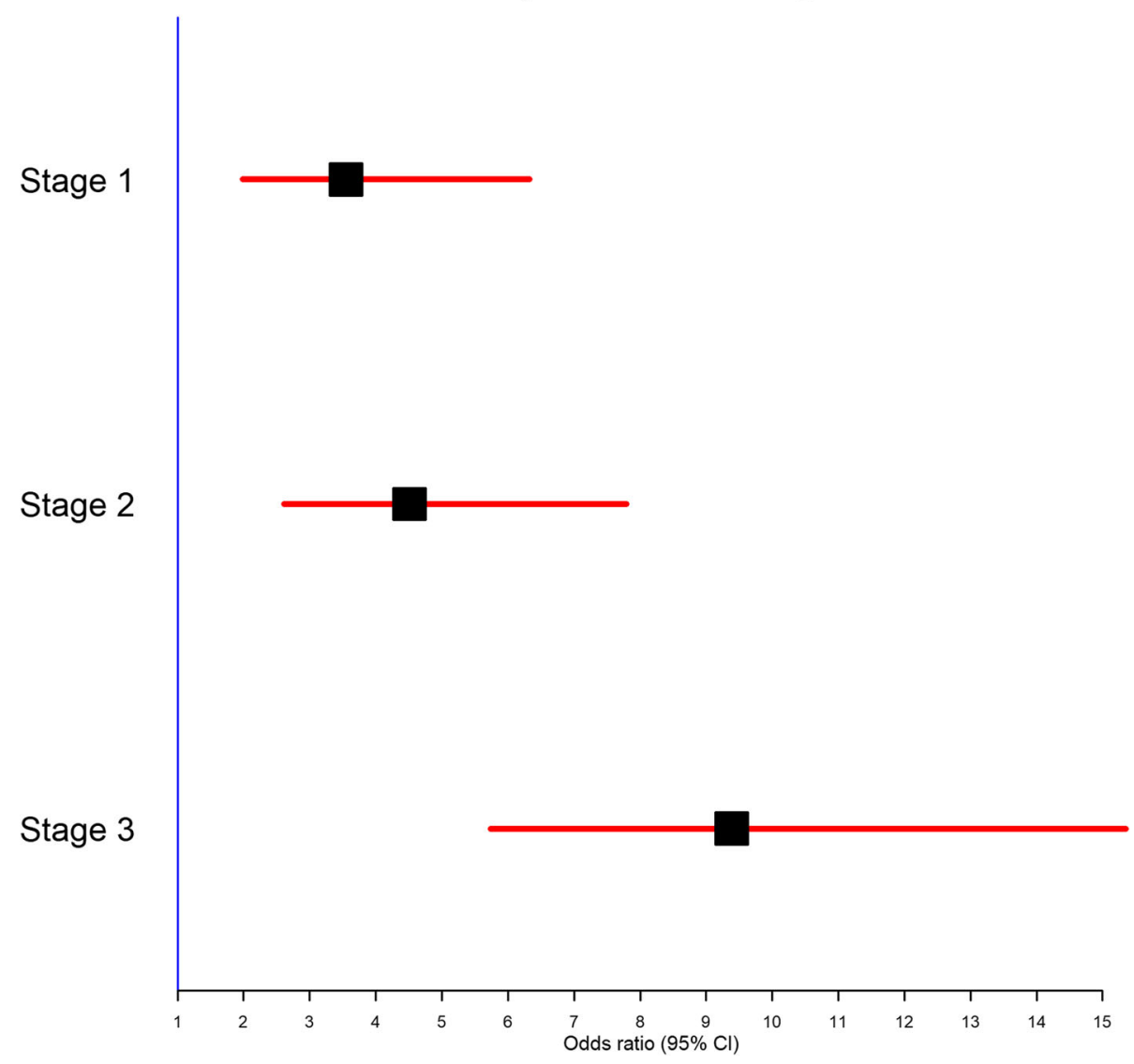

Fig. 5 The odds of dying for increasing KDIGO stage of severity

Table 3 Multivariate analysis for predictors of mortality in the AKI cohort ( $n=497)$

\begin{tabular}{llll}
\hline Covariate & Crude odds ratio $(95 \% \mathrm{Cl})$ & Adjusted odds ratio $(95 \% \mathrm{Cl})$ & $p$-value \\
\hline Age, per 10 year increase & $1.16(1.030 ; 1.300)$ & $1.33(1.12 ; 1.59)$ & 0.002 \\
Gender (male) & $0.88(0.596 ; 1.296)$ & $0.96(0.584 ; 1.588)$ & 0.878 \\
Diabetes & $0.8(0.462 ; 1.348)$ & $0.47(0.223 ; 0.963)$ & 0.043 \\
Active malignancy & $0.82(0.259 ; 2.218)$ & $1.03(0.25 ; 3.782)$ & 0.963 \\
Active tuberculosis & $2.14(1.042 ; 4.370)$ & $2.84(1.14 ; 7.079)$ & 0.025 \\
CKD & $0.87(0.372 ; 1.880)$ & $1.12(0.357 ; 3.331)$ & 0.840 \\
Hypertension & $1.1(0.733 ; 1.665)$ & $1.47(0.790 ; 2.81)$ & 0.224 \\
Receiving HAART & $0.99(0.585 ; 1.719)$ & $1.37(0.670 ; 2.88)$ & 0.398 \\
SAPS 3 score & $1.07(1.054 ; 1.089)$ & $1.04(1.026 ; 1.065)$ & $<0.001$ \\
ICU days & $0.99(0.971 ; 1.010)$ & $0.96(0.929 ; 0.985)$ & 0.004 \\
Mechanical ventilation & $5.00(3.087 ; 8.420)$ & $2.08(1.084 ; 4.062)$ & 0.029 \\
Requiring vasopressors & $7.26(4.782 ; 11.182)$ & $3.99(2.318 ; 6.961)$ & $<0.001$ \\
ARDS & $1.43(0.678 ; 2.930$ & $0.82(0.335 ; 1.973)$ & 0.661 \\
Sepsis & $3.15(2.127 ; 4.686)$ & $1.8(1.047 ; 3.083)$ & 0.033 \\
HIV status ${ }^{a}$ & $1.76(1.05 ; 2.94)$ & $1.11(0.56 ; 2.15)$ & 0.769 \\
\hline
\end{tabular}

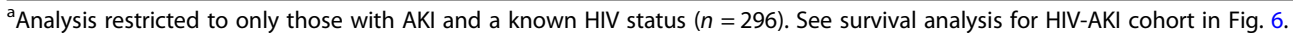




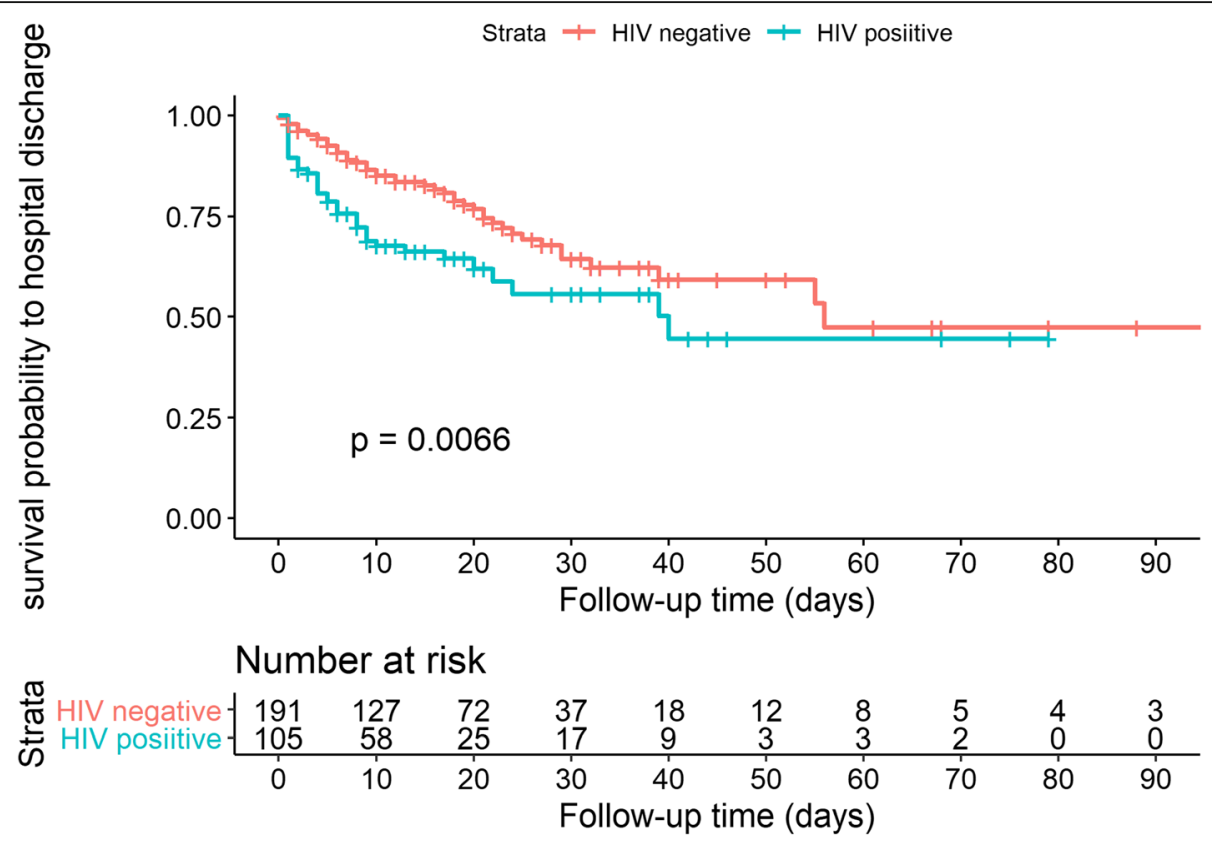

Fig. 6 Kaplan Meier plot showing the association between survival probability by HIV status for those with AKI, Hazard ratio $(95 \% \mathrm{Cl})=1.8(1.18$; 2.74). The analysis was restricted to only those with AKI and a known HIV status $(n=296)$

\section{Renal recovery from AKI}

Functional renal recovery was assessed at 90 days after hospital discharge in patients with AKI who survived $(n=332)$ : Full recovery occurred in 236 (100\%) of those who developed maximum KDIGO stage 1 or 2 AKI and 96 of 110 (87.3\%) who developed stage 3 AKI. CKD was therefore established in 14 patients (12.7\% of survivors) with a median creatinine (IQR) of $175(137 ; 424) \mu \mathrm{mol} / \mathrm{L}$. Non-recovery of renal function in survivors was associated with a higher admission creatinine (670 vs $257 \mu \mathrm{mol} / \mathrm{L} ; p$-value 0.004$)$ and receiving dialysis $(64.3 \%$ vs $32.3 \%, p=0.043)$, but not with admission severity score (SAPS 3 admission score $=55.1$ versus 56.1, $p=0.811$ ), premorbid creatinine values (76 $\mu \mathrm{mol} / \mathrm{L} ; \mathrm{IQR} 60 ; 228$ versus $76 \mathrm{umol} / \mathrm{L} ; \mathrm{IQR} 56 ; 130$, $p=0.796)$ nor receiving prolonged dialysis modalities like SLEDD and CRRT (44.4\% versus $29.03 \%$ respectively, $p=$ $0.667)$. The group that did not recover was less likely to have pre-renal failure $(14.3 \%$ versus $51.0 \%, p=0.022)$ as a cause for AKI. This group also tended to have more diabetes $(35.7 \%$ vs $15.6 \%, p=0.069)$ and hypertension $(57.1 \%$ vs $31.3 \%, p=0.057$ ), a lower CD4 count if HIV positive (55, IQR 45; 65, vs 165, IQR 131; 312; $p=0.067$ ) and a higher HIV viral $\operatorname{load}(\log 4.06$ vs $\log 1.94, p=0.247)$. HIV infection itself and the receipt of HAART was not associated with non-recovery of renal function in survivors, $p=$ 0.972 and 0.886 respectively. No patients were dialysis dependent at 90-days.

\section{Other differences between HIV positive and negative patients with AKI}

HIV positive patients with AKI were more likely to be of Black African ethnicity ( $85.7 \%$ vs $59.2 \%, p<0.001)$, to be an emergency admission (92.4\% vs $23.0 \%, \mathrm{p}<0.001)$, to have a medical rather than surgical problem $(73.3 \%$ vs $26.2 \%, \mathrm{p}<0.001)$, to have sepsis $(41.3 \%$ vs $32.1 \%, p=$ $0.049)$ and to have active tuberculosis $(15.5 \%$ vs $6.85 \%$, $p<0.003)$.

\section{Discussion}

This is the largest prospective African study of AKI in critically ill adults with an HIV seropositive rate of $32.6 \%$. Similar studies include reports from Morocco $(n=97)$ [29], the Democratic Republic of Congo (DRC, $n=476)$ [30] and Egypt $(n=532)$ [31]. However, only the DRC study reported HIV prevalence, which was low at $2.9 \%$. Consistent with other LMI countries, patients were younger than in $\mathrm{HI}$ country cohorts (mean age 40.6 years) with lower comorbidity rates [1, 18, 19, 29-32], mostly of Black African ethnicity, and were representative of the local community that we serve [21].

As in other African [29-31] and HI country [1] ICUbased studies, AKI was very common in our cohort and affected nearly two thirds (58.5\%) of all patients admitted to the ICU. AKI was associated with male gender, higher severity of illness, more sepsis, longer ICU stay and the need for vasopressors. Pre-existing CKD was 
negatively associated with AKI but reflects an admission bias against very ill patients with CKD to the unit due to a lack of resources to continue with chronic renal replacement therapy in most. Increased age, severity of illness, sepsis, mechanical ventilation, the use of vasopressors and the presence of tuberculosis were independently associated with mortality in those with AKI. Furthermore, increasing stage of AKI showed a stepwise increase in the risk of mortality.

Although our unit admits emergency medical as well as elective surgical cases, just over a quarter of all admissions were trauma related, reflecting the alarmingly high levels of interpersonal violence and road traffic accidents prevalent in South Africa. In 2010, interpersonal violence and road injury combined were the second leading cause of death and disability adjusted life years lost in South Africa, after HIV which was the leading cause [33]. Consequently, a large proportion of AKI was attributable to hypovolaemic shock (55.1\%) and rhabdomyolysis $(24.8 \%)$ in keeping with the degree of trauma-related admissions. Two recent retrospective studies in South Africa also highlighted AKI in trauma victims as a major contributor to morbidity and mortality [34,35]. This reflects a major change in the epidemiology of AKI in South Africa where older reports have not highlighted trauma as a major cause of AKI $[14,15,36]$ and has important public health implications for health administrators. Sepsis was also a common precipitant of AKI at $40.6 \%$ which is similar to that reported in other LMI country studies in the critically ill $[1,31,37]$. Herbal and traditional medicine use is not a prominent cause of AKI in our region compared with prior reports from other regions $[38,39]$. Tropical diseases such as malaria are not endemic in our region and are therefore also not common precipitants of AKI.

Severe AKI (KDIGO stage 3) was common, affecting $41.4 \%$ of all those with AKI and $24.4 \%$ of all admissions. The number of patients dialyzed $(10.4 \%$ of all admissions) was slightly lower than in the large Acute Kidney Injury-Epidemiologic Prospective Investigation (AKIEPI) study where $13.5 \%$ of all admissions were dialyzed [1] and may be explained by local practice to usually delay the initiation of dialysis until more classic indications exist. Notwithstanding this fact, most patients requiring dialysis were initiated early (within $24 \mathrm{~h}$ of ICU admission) reflecting the advanced state of organ dysfunction at admission and late presentation that is common in LMI countries [32]. Although the AKI-EPI study was multinational, only 34 patients from Africa were included. Continuous renal replacement therapies are available in our center but cost in excess of 10 times more than intermittent dialysis. As such, CRRT is reserved for specific indications and the rate of CRRT use was much lower in our study at $13.6 \%$ compared to $75.2 \%$ in the
AKI-EPI study. Of those who developed AKI and survived, a significant proportion of patients with stage 3 AKI did not recover renal function fully (12.7\%).

South Africa has a very high HIV burden of 7.9 million people living with HIV as well as the largest number of people on antiretroviral therapy in the world [40]. The HIV seropositive rate of $32.6 \%$ in our cohort is consistent with the known background prevalence of HIV in our province of $25.2 \%$ in adults aged 15-49 when measured in 2018 [40]. This is vastly different to a retrospective study in a South African medical ICU in 2004 [41] where only three patients (6.5\%) in the AKI cohort were HIV positive. In our study, active tuberculosis was very common, affecting 1 in 16 of all admissions. This is likely in part due to the high prevalence of HIV, but also due to the high reported background incidence of tuberculosis in the community of 1095 cases/100 000 per year [42]. Whilst tuberculosis was not associated with AKI per se, it was independently associated with mortality. Many cases of active tuberculosis were diagnosed during ICU admission through microbiological means and many were not the primary cause of admission.

HIV infection was associated with higher mortality, as well as the presence of sepsis and active tuberculosis. It was also associated with increased severity of illness and the need for emergency admission. Length of stay was shorter for those with HIV, but reflects earlier mortality in those with increased severity of illness. As in other studies in the post-HAART era [36, 37, 43-45], it would appear from our data that traditional predictors of mortality such as higher severity of illness are implicated in predicting mortality and not HIV status, CD4 count, viral suppression nor the use of HAART.

Proportionally few (22.9\%) HIV positive patients that developed AKI were receiving Tenofovir-based HAART at the time of ICU admission. Although there was little difference in viral suppression between groups, the CD4 count was significantly lower in the group that developed AKI thereby placing patients at risk for the immune reconstitution inflammatory syndrome (IRIS) [46]. While tenofovir has been shown to be nephrotoxic [47], we hypothesize that the pathogenesis of AKI in at least some of our HIV patients was related to the often recent initiation of HAART with the development of an unmasking tuberculosis-associated IRIS [48] and consequent tuberculosis sepsis syndrome with associated AKI. This has previously been reported [49] and is likely to be under-recognized.

\section{Study limitations and strengths}

This study needs to be viewed in the context of its limitations. We may have underestimated the incidence of AKI since we were unable to reliably utilize the KDIGO urine output criterion for diagnosis as patients admitted 
to the ICU were not always weighed or catheterised. Secondly, $43.9 \%$ of all patients admitted to the ICU were not tested for HIV. All patients who were able to consent were encouraged to have an HIV test; however, patients that were moribund or confused were not tested for HIV without indication or consent. On the other hand, to our knowledge, this is the largest published prospective cohort of critically ill adults with HIV and AKI [50]. The study was inclusive of all major disciplines with the exception of cardiothoracics and the loss to follow up was low at $1.4 \%$. Standardised criteria for the diagnosis of all stages of AKI were used and 90-day renal recovery data was obtained.

\section{Conclusion}

In this large prospective multidisciplinary ICU cohort of younger patients in a LMI country with a high HIV prevalence and many trauma related admissions, AKI was frequently encountered, and was associated with a high mortality, but good functional renal recovery in most survivors. While HIV infection was associated with higher mortality, this was due to increased severity of illness, not HIV status per se.

\section{Abbreviations}

AKI: Acute Kidney Injury; ARDS: Acute Respiratory Distress Syndrome; CD4: Cluster Differentiation 4; CKD: Chronic Kidney Disease: CRRT: Continuous Renal Replacement Therapy; eGFR: Estimated Glomerular Filtration Rate; HAART: Highly Active Antiretroviral Therapy; HI: High Income; HIV: Human Immunodeficiency Virus; ICU: Intensive Care Unit; IHD: Intermittent Haemodialysis; IQR: Interquartile Range; IRIS: Immune Reconstitution Inflammatory Syndrome; IV: Intravenous; KDIGO: Kidney Diseases Improving Global Outcomes; LMI: Low-middle Income; REDCap: Research Electronic Data Capture; SAPS: Simplified Acute Physiology Score; SD: Standard Deviation; SLEDD: Sustained Low Efficiency Daily Dialysis; SOFA: Sequential Organ Failure Assessment

\section{Acknowledgements}

We would like to thank all the rotating doctors who helped collect data. Noline van Vuuren was invaluable in data checking and cleaning.

\section{Authors' contributions}

RA wrote the protocol, collected and cleaned data and wrote the manuscript. EvdM assisted with the protocol, consultancy and manuscript writing. SP cleaned data and performed the statistical analysis. MvN collected and cleaned data. JE assisted with consultancy, following up patients in the renal unit and appraisal of the final manuscript. DB assisted with data collection, consultancy and appraisal of the final manuscript. RF assisted with the protocol, consultancy, manuscript writing and follow up of patients in the renal unit. All authors approved the final version of the manuscript.

\section{Funding}

The authors gratefully acknowledge Roche South Africa for an unrestricted research grant that was used to fund data capturing, analysis, presentation and publication of the study (REF: SA/NONP/1812/0050). The funder did not contribute to the design, conduct of the study, analysis or interpretation of results.

\section{Availability of data and materials}

Datasets are accessible from the Mendeley Data public repository available at https://doi.org/10.17632/7f6yxz2d4c.1
Ethics approval and consent to participate

Ethical approval was granted by the Human Research Ethics Committee of Walter Sisulu University (protocol number 067/2016). Participant consent was waived by the Committee.

\section{Consent for publication}

Not applicable.

\section{Competing interests}

The authors declare that they have no competing interests.

\section{Author details}

${ }^{1}$ Adult Critical Care Unit, Livingstone Hospital, Port Elizabeth, South Africa. ${ }^{2}$ Walter Sisulu University, Mthatha, South Africa. ${ }^{3}$ Department of Statistics, Nelson Mandela University, Port Elizabeth, South Africa. ${ }^{4}$ Division of Nephrology and Hypertension, Livingstone Hospital, Port Elizabeth, South Africa. ${ }^{5}$ Department of Medicine, Division Nephrology and Hypertension, University of Cape Town, Cape Town, South Africa.

Received: 15 May 2019 Accepted: 8 November 2019

Published online: 10 December 2019

\section{References}

1. Hoste EA, Bagshaw SM, Bellomo R, et al. Epidemiology of acute kidney injury in critically ill patients: the multinational AKI-EPI study. Intensive Care Med. 2015;41:1411-23.

2. Koeze J, Keus F, Dieperink W, van der Horst ICC, ZijIstra JG, van Meurs M. Incidence, timing and outcome of AKI in critically ill patients varies with the definition used and the addition of urine output criteria. BMC Nephrol. 2017:18:70.

3. Zeng X, McMahon GM, Brunelli SM, Bates DW, Waikar SS. Incidence, outcomes, and comparisons across definitions of AKI in hospitalized individuals. Clin J Am Soc Nephrol. 2014;9:12-20.

4. Linder A, Fjell C, Levin A, Walley KR, Russell JA, Boyd JH. Small acute increases in serum creatinine are associated with decreased long-term survival in the critically ill. Am J Respir Crit Care Med. 2014;189:1075-81.

5. Lameire NH, Bagga A, Cruz D, et al. Acute kidney injury: an increasing global concern. Lancet. 2013;382:170-9.

6. Lewington AJ, Cerdá J, Mehta RL. Raising awareness of acute kidney injury: a global perspective of a silent killer. Kidney Int. 2013;84:457-67.

7. Gammelager H, Christiansen CF, Johansen MB, Tonnesen E, Jespersen B, Sorensen HT. Five-year risk of end-stage renal disease among intensive care patients surviving dialysis-requiring acute kidney injury: a nationwide cohort study. Crit Care. 2013;17:R145.

8. Chawla LS, Eggers PW, Star RA, Kimmel PL. Acute kidney injury and chronic kidney disease as interconnected syndromes. N Engl J Med. 2014;371:58-66.

9. Delannoy B, Floccard B, Thiolliere F, et al. Six-month outcome in acute kidney injury requiring renal replacement therapy in the ICU: a multicentre prospective study. Intensive Care Med. 2009;35:1907-15.

10. Villeneuve PM, Clark EG, Sikora L, Sood MM, Bagshaw SM. Health-related quality-of-life among survivors of acute kidney injury in the intensive care unit: a systematic review. Intensive Care Med. 2016:42:137-46.

11. Pickkers $P$, Ostermann $M$, Joannidis $M$, et al. The intensive care medicine agenda on acute kidney injury. Intensive Care Med. 2017:43:1198-209.

12. Investigators RRTS. Intensity of continuous renal-replacement therapy in critically ill patients. N Engl J Med. 2009;361:1627-38.

13. Network VNARFT. Intensity of renal support in critically ill patients with acute kidney injury. N Engl J Med. 2008:359:7-20.

14. Naicker S, Aboud O, Gharbi MB. Epidemiology of acute kidney injury in Africa. Semin Nephrol. 2008;28(4):348-53.

15. Swanepoel CR, Wearne N, Okpechi IG. Nephrology in Africa-not yet uhuru. Nat Rev Nephrol. 2013;9:610.

16. Hobson C, Ozrazgat-Baslanti T, Kuxhausen A, et al. Cost and mortality associated with postoperative acute kidney injury. Ann Surg. 2015;261:1207-14.

17. Moosa MR, Meyers AM, Gottlich E, Naicker S. An effective approach to chronic kidney disease in South Africa. South African medical journal =. Suid-Afrikaanse tydskrif vir geneeskunde. 2016;106:156-9.

18. Bagasha P, Nakwagala F, Kwizera A, Ssekasanvu E, Kalyesubula R. Acute kidney injury among adult patients with sepsis in a low-income country: clinical patterns and short-term outcomes. BMC Nephrol. 2015;16:4. 
19. Kwizera A, Tumukunde J, Ssemogerere L, et al. Clinical characteristics and 30-day outcomes of intermittent hemodialysis for acute kidney injury in an African intensive care unit. Biomed Res Int. 2016;2016:6. Article ID 2015251. https://doi.org/10.1155/2016/2015251.

20. Mehta RL, Cerda J, Burdmann EA, et al. International Society of Nephrology's 0by25 initiative for acute kidney injury (zero preventable deaths by 2025): a human rights case for nephrology. Lancet (London, England). 2015;385: 2616-43.

21. Africa SS. Census 2011 Provincial Profile: Eastern Cape. 2014.

22. Joynt GM, Gopalan DP, Argent AA, et al. The Critical Care Society of Southern Africa Consensus Guideline on ICU Triage and Rationing (ConlCTri). 2019.

23. Palevsky PM, Liu KD, Brophy PD, et al. KDOQI US commentary on the 2012 KDIGO clinical practice guideline for acute kidney injury. Am J Kidney Dis. 2013;61:649-72

24. Siew ED, Matheny ME. Choice of reference serum Creatinine in defining acute kidney injury. Nephron. 2015;131:107-12

25. Moreno RP, Metnitz PG, Almeida E, et al. SAPS 3--from evaluation of the patient to evaluation of the intensive care unit. Part 2: development of a prognostic model for hospital mortality at ICU admission. Intensive Care Med. 2005;31:1345-55.

26. Vincent JL, Moreno R, Takala J, et al. The SOFA (Sepsis-related organ failure assessment) score to describe organ dysfunction/failure. On behalf of the working group on Sepsis-related problems of the European Society of Intensive Care Medicine. Intensive Care Med. 1996;22:707-10.

27. Shankar-Hari M, Phillips GS, Levy ML, et al. Developing a new definition and assessing new clinical criteria for septic shock: for the third international consensus definitions for Sepsis and septic shock (Sepsis-3). JAMA. 2016;315: 775-87.

28. Harris PA, Taylor R, Thielke R, Payne J, Gonzalez N, Conde JG. Research electronic data capture (REDCap)--a metadata-driven methodology and workflow process for providing translational research informatics support. J Biomed Inform. 2009;42:377-81.

29. Nechba RB, El M'barki K, Mesfioui A, Zeggwagh AA. Epidemiology of acute kidney injury in Moroccan medical intensive care patients: a regional prospective, observational study. Sci J Public Health. 2014;2:1-6.

30. Masewu A, Makulo J-R, Lepira F, et al. Acute kidney injury is a powerful independent predictor of mortality in critically ill patients: a multicenter prospective cohort study from Kinshasa, the Democratic Republic of Congo. BMC Nephrol. 2016;17:118.

31. ElHafeez SA, Tripepi G, Quinn R, et al. Risk, predictors, and outcomes of acute kidney injury in patients admitted to intensive care units in Egypt. Sci Rep. 2017;7:17163.

32. Cerdá J, Bagga A, Kher V, Chakravarthi RM. The contrasting characteristics of acute kidney injury in developed and developing countries. Nat Rev Nephrol. 2008:4:138.

33. Mayosi BM, Benatar SR. Health and health care in South Africa-20 years after Mandela. N Engl J Med. 2014;371:1344-53.

34. Skinner DL, Kong VY, de Vasconcellos $K$, et al. Acute kidney injury on presentation to a major trauma service is associated with poor outcomes. J Surg Res. 2018;232:376-82.

35. Dlamini TA, Heering PJ, Chivese T, Rayner B. A prospective study of the demographics, management and outcome of patients with acute kidney injury in Cape Town, South Africa. PLoS One. 2017;12:e0177460.

36. Vachiat Al, Musenge E, Wadee S, Naicker S. Renal failure in HIV-positive patients—a South African experience. Clin Kidney J. 2013;6:584-9.

37. Arendse C, Okpechi I, Swanepoel C. Acute dialysis in HIV-positive patients in Cape Town, South Africa. Nephrology. 2011;16:39-44.

38. Swanepoel C, Blockman M, Talmud J. Nephrotoxins in Africa. In: De Broe ME, Porter GA, Bennett WM, Deray G, editors. Clinical Nephrotoxins: renal injury from drugs and chemicals. Boston: Springer US; 2008. p. 859-70.

39. Luyckx VA, Naicker S. Acute kidney injury associated with the use of traditional medicines. Nat Clin Pract Nephrol. 2008;4:664.

40. (HSRC) HSRC. The fifth South African national hiv prevalence, incidence, behaviour and communication survey, 2017 (SABSSM V1).

41. Friedericksen DV, Van der Merwe L, Hattingh TL, Nel DG, Moosa MR. Acute renal failure in the medical ICU still predictive of high mortality. South African medical journal =. Suid-Afrikaanse tydskrif vir geneeskunde. 2009;99: 873-5.
42. Nanoo A, Izu A, Ismail NA, et al. Nationwide and regional incidence of microbiologically confirmed pulmonary tuberculosis in South Africa, 200412: a time series analysis. Lancet Infect Dis. 2015;15:1066-76.

43. Randall DW, Brima N, Walker D, et al. Acute kidney injury among HIVinfected patients admitted to the intensive care unit. Int J STD AIDS. 2015; 26:915-21.

44. Mkoko P, Raine RI. HIV-positive patients in the intensive care unit: a retrospective audit. South African medical journal $=$. Suid-Afrikaanse tydskrif vir geneeskunde. 2017;107:877-81.

45. Luna L, Soares D, Silva J, et al. Clinical characteristics, outcomes and risk factors for death among critically ill patients with HIV-related acute kidney injury. Rev Inst Med Trop Sao Paulo. 2016;58:52. http://dx.doi.org/10.1590/ S1678-9946201658052

46. Meintjes G, Sonderup MW. A practical approach to the diagnosis and management of paradoxical tuberculosis immune reconstitution inflammatory syndrome. Continuing Med Educt. 2011;29(10):410-417.

47. Herlitz LC, Mohan S, Stokes MB, Radhakrishnan J, D'Agati VD, Markowitz GS. Tenofovir nephrotoxicity: acute tubular necrosis with distinctive clinical, pathological, and mitochondrial abnormalities. Kidney Int. 2010;78:1171-7.

48. Meintjes G, Rabie H, Wilkinson RJ, Cotton MF. Tuberculosis-associated immune reconstitution inflammatory syndrome and unmasking of tuberculosis by antiretroviral therapy. Clin Chest Med. 2009:30:797-810.

49. Salliot C, Guichard I, Daugas E, Lagrange M, Verine J, Molina J-M. Acute kidney disease due to immune reconstitution inflammatory syndrome in an HIV-infected patient with tuberculosis. J Int Assoc Physicians AIDS Care. 2008;7:178-81

50. Nel J, Moosa M. The outcome of HIV-positive patients admitted to intensive care units with acute kidney injury. Renal Failure-The Facts: InTech; 2012.

\section{Publisher's Note}

Springer Nature remains neutral with regard to jurisdictional claims in published maps and institutional affiliations.

Ready to submit your research? Choose BMC and benefit from:

- fast, convenient online submission

- thorough peer review by experienced researchers in your field

- rapid publication on acceptance

- support for research data, including large and complex data types

- gold Open Access which fosters wider collaboration and increased citations

- maximum visibility for your research: over $100 \mathrm{M}$ website views per year

At $\mathrm{BMC}$, research is always in progress.

Learn more biomedcentral.com/submissions 